\title{
TOXICIDAD AGUDA DE LAS HOJAS DE Xanthium spinosum EN RATONES BALB/C
}

\author{
Arturo Silvero-Isidre ${ }^{1, a}$, Sergio Morínigo-Guayuán ${ }^{1, a}$, Aaron Meza-Ojeda',a ${ }^{1,}$ Marcelo Mongelós-Cardozo ${ }^{1, a}$, \\ Aldo González-Ayalaª,b, Susy Figueredo-Thiele,c
}

\begin{abstract}
RESUMEN
Las hojas de la planta Xanthium spinosum se utilizan culturalmente en Paraguay por sus propiedades medicinales. Se evaluó la toxicidad aguda del extracto de hojas maduras; para el estudio se seleccionaron 35 ratones BALB/C machos que fueron distribuidos en 7 grupos, 6 de ensayo y 1 de control. El extracto fue preparado en concentraciones de 6 y $9 \%$ (g/dL); se administró la solución $6 \%$ a tres grupos y la solución $9 \%$ a los otros tres grupos, con dosis entre 200 y $1000 \mathrm{mg} / \mathrm{kg}$. Al final de 14 días de observación, se extrajeron muestras de sangre para estudios laboratoriales de urea y transaminasas, además de órganos para estudios anatomopatológicos. Se determinaron aumentos en los niveles de GOT y urea en comparación al grupo control. Se concluye que el consumo del extracto de hojas maduras de Xanthium spinosum puede causar daño hepático.
\end{abstract}

Palabras clave: Xanthium spinosum; Toxicidad aguda; Plantas medicinales (fuente: DeCS BIREME).

\section{ACUTE TOXICITY OF Xanthium spinosum LEAVES IN BALB/C MICE}

\begin{abstract}
The leaves of the Xanthium spinosum plant have been used culturally in Paraguay for their medicinal properties. Acute toxicity of mature leaf extract was evaluated. For the study, 35 Balb/c mice were selected and allocated into 7 groups, 6 test groups and 1 control group. The extract was prepared in concentrations of $6 \%$ and $9 \%(\mathrm{~g} / \mathrm{dL})$. The $6 \%$ concentration was administrated to 3 test groups and 9\% concentration to the remaining 3 groups, with doses between 200 and $1000 \mathrm{mg} / \mathrm{kg}$ per mouse. After 14 days of observation, blood samples were taken for laboratory studies of urea and transaminases and organs were examined for pathological studies. There were increased levels of GOT and urea in the test groups compared to the control group. In conclusion, the consumption of mature leaf extract of Xanthium spinosum can cause hepatic damage.
\end{abstract}

Key words: Xanthium spinosum; Acute toxicity; Plants, medicinal (source: MeSH NLM).

\section{INTRODUCCIÓN}

Desde hace siglos, diversas culturas han utilizado diferentes especies vegetales alegando propiedades medicinales, una de estas, con gran influencia en América fue la de los indígenas guaraníes. El conocimiento que tenían de las especies vegetales era tan extenso y profundo, y su lengua es una de las que más términos ha aportado a la nomenclatura botánica, así, en la actualidad, en los diccionarios y vocabularios científicos paraguayos se registran más de 700 géneros y más de cuarenta familias botánicas de origen guaraní ${ }^{(1)}$.

Xanthium spinosum var Spinosum de nombre popular "cepacaballo" es una hierba que pertenece a la familia Asterácea, originaria de las zonas cálido-templadas de Sudamérica. Se la utiliza por sus propiedades medicinales; la parte aérea es conocida por sus propiedades diuréticas y para combatir las infecciones; la raíz como depurativo, febrífugo, diurético, para crear repugnancia al alcohol, y en afecciones hepáticas y urinarias ${ }^{(2)}$

En cuanto a los efectos tóxicos, la literatura registra algunos estudios sobre la genotoxicidad de Xanthium spinosum (3), sin embargo, se ha estudiado más extensamente la especie Xanthium strumariun, aunque cabe destacar que estas especies vegetales tienen el mayor grado de parentesco en la familia. En el caso de Xanthium strumariaun, se han reportado efectos tóxicos, desencadenando hipoglicemia, daño hepático y convulsiones ${ }^{(4)}$.

Los componentes tóxicos se encuentran concentrados en las semillas y los brotes, aunque también se han constatado efectos con el consumo de plantas maduras ${ }^{(5)}$. Esta propiedad

\footnotetext{
Facultad de Ciencias Médicas. Universidad Nacional de Asunción. Asunción, Paraguay.

2 Departamento de Medicina Tropical. Instituto de Investigaciones en Ciencias de la Salud. Universidad Nacional de Asunción. Asunción, Paraguay.

3 Departamento de Patología. Instituto de Investigaciones en Ciencias de la Salud. Universidad Nacional de Asunción. Asunción, Paraguay.

a Estudiante de Medicina; ${ }^{\mathrm{b}}$ bioquímico; ${ }^{\mathrm{c}}$ médica patóloga

Recibido: 21-05-15 Aprobado: 07-10-15
}

Citar como: Silvero-Isidre A, Morínigo-Guayuán S, Meza-Ojeda A, Mongelós-Cardozo M, González-Ayala A, Figueredo-Thiel S. Toxicidad aguda de las hojas de Xanthium spinosum en ratones BALB/C. Rev Peru Med Exp Salud Publica. 2016;33(1):113-9.doi: 10.17843/rpmesp.2016.331.1888 
tiene relevancia en el momento de la utilización de los extractos, pues supone inconvenientes en el desarrollo de los protocolos, produciendo efectos tóxicos antes de la conclusión de la investigación de sus propiedades ${ }^{(6)}$. La aplicación de las plantas medicinales debe efectuarse sobre una base científica que valide la efectividad terapéutica y su relativa inocuidad ${ }^{(7)}$.

Alrededor del $80 \%$ de la población mundial recurre a la medicina tradicional herbolaria para la atención primaria de la salud. Varias farmacias las procesan como jarabes, pastillas y otros compuestos obtenidos en forma natural. La demanda de estos productos se encuentra en aumento tanto en el Paraguay como en los otros países de la región, que tienden hacia el consumo de medicinas alternativas $^{(8)}$. Ante esta realidad, los estudios sobre las diferentes especies vegetales cobran importancia, y antes de conocer sus propiedades beneficiosas, sería prioridad el control de sus propiedades toxicológicas para evitar efectos adversos. El objetivo del estudio es indagar sobre el posible efecto tóxico del consumo de hojas de plantas maduras de Xanthium spinosum en ratones $\mathrm{BALB} / \mathrm{c}$.

\section{MATERIALES Y MÉTODOS}

\section{DISEÑO}

Estudio de diseño experimental de ensayo de campo con muestreo aleatorio, probabilístico realizado en la Facultad de Ciencias Médicas de la Universidad Nacional de Asunción (FCM-UNA) y en el Instituto de Investigaciones en Ciencias de la Salud de la Universidad Nacional de Asunción (IICS-UNA), de septiembre a octubre de 2012.

\section{IDENTIFICACIÓN Y RECOLECCIÓN DE LA ESPECIE VEGETAL}

La especie vegetal fue adquirida en el Mercado Municipal N. ${ }^{\circ} 4$ (Asunción, Paraguay), de un puesto dedicado a la comercialización de plantas medicinales, recolectadas en la ciudad de Acahay (25 $5^{\circ} 54^{\prime}$ 56.16" latitud sur, $56^{\circ} 50^{\prime} 26.88^{\prime \prime}$ longitud oeste), distrito del departamento de Paraguarí, Paraguay. La identificación botánica correspondió al Departamento de Botánica de la Facultad de Ciencias Químicas, son referencia ejemplar de herbario CC \& GG 164, FM 1678.

\section{ELABORACIÓN DEL EXTRACTO DE HOJAS}

Fueron separadas las raíces y las hojas de la planta, y secadas en una estufa con recirculación de aire a $50{ }^{\circ} \mathrm{C}$ durante 7 días en el Departamento de Botánica de la Facultad de Ciencias Químicas de la Universidad
Nacional de Asunción. Se realizó el extracto etanólico, macerado en frío, de Xanthium spinosum $L$. var. spinosum elaborado en el Departamento de Medicina Tropical del Instituto de Investigaciones en Ciencias de la Salud. Las muestras de ensayo se prepararon a partir del concentrado obtenido de la destilación con rotavapor.

\section{DISTRIBUCIÓN DE LOS ANIMALES}

Se utilizaron ratones BALB/c machos adultos jóvenes (7-8 semanas) con un peso promedio de 19,87 g, procedentes del Instituto de Investigaciones de la Universidad Nacional de Asunción (IICS-UNA), los animales tuvieron un tiempo de aclimatación a las condiciones del laboratorio de 3 días. La temperatura se mantuvo entre $28 \pm 3^{\circ} \mathrm{C}$, y los ciclos de luz-oscuridad fueron de $12 \mathrm{~h}$. La dieta proporcionada fue conforme a la American Institute of Nutrition 1993 (AIN-93 G), junto con agua ad libitum. Se cambiaron las virutas de madera dos veces a la semana.

Se estableció un grupo control negativo con cinco animales y dos grupos de ensayo de quince animales, de acuerdo a la concentración de extracto que les fue administrado (grupos I, y II; concentraciones de extracto 6 y $9 \%(\mathrm{~g} / \mathrm{mL})$ respectivamente). Cada uno de estos grupos, a su vez, fue dividido en tres subgrupos $(A, B, C)$, de acuerdo con la dosis que se suministra a cada animal (200, 500 y $1000 \mathrm{mg} / \mathrm{kg}$ de peso respectivamente). La distribución de los animales se realizó al azar. Para mejorar la disolución, el extracto fue diluido en solución acuosa con tween $80^{\circledR}$ al $1 \%$, el grupo control consumió solución acuosa con tween $80^{\circledR}$ al $1 \%$. La dosis fue administrada por vía oral, por medio de una cánula intragástrica.

\section{EVALUACIÓN DIARIA}

Se realizó una observación diaria en el horario comprendido entre las 11.00-12.30 h durante los 14 días posteriores a la administración. Durante cada observación se tuvo en cuenta el estado clínico general del animal de acuerdo con los siguientes parámetros: piloerección, presencia de temblores, alteración de mucosa ocular, falta de movilidad, caída del pelo, lesiones o muerte.

\section{EXTRACCIÓN DE ÓRGANOS Y SANGRE}

Al momento del sacrificio en cámara de $\mathrm{CO}_{2}$, se realizó la punción cardiaca para la recolección de sangre y la extracción de hígado, riñones, bazo y corazón a cada ratón, por medio de la utilización de kit de disección. Se procedió al análisis laboratorial de GOT (transaminasa glutámico-oxalacética) y GPT (transaminasa glutámicopirúvica) por métodos cinéticos y la urea por métodos enzimáticos. 


\section{EVALUACIÓN ANATOMOPATOLÓGICA}

Se evaluó el aspecto macroscópico (peso y color) de los órganos que luego fueron conservados en formol al $10 \%$. Los cortes representativos de los diversos órganos se incluyeron en parafina y se seccionaron con micrótomo a un grosor de 4 a $5 \mu \mathrm{m}$. Se utilizó la técnica de coloración de hematoxilina-eosina que se realizó en el Departamento de Patología del IICS-UNA. Los indicadores de toxicidad fueron edema, acumulaciones intracelulares, congestión vascular, necrosis, y hemorragias.

\section{EXTRAPOLACIÓN DE DATOS}

En la extrapolación de la dosis se consideró la superficie corporal total, según la FDA Draft Guidelines ${ }^{(9)}$.

\section{CONSIDERACIONES ÉTICAS}

Una vez comprobada la escasa cantidad de estudios precedentes y la ausencia de modelos de simulación, el empleo de animales fue indispensable para llevar a cabo este ensayo. Se cumplieron con las recomendaciones del Consejo Canadiense de Protección de Animales en los aspectos de suministro de alimentos y limpieza de cajas ${ }^{(10)}$. El proyecto del estudio contó con la aprobación del Comité de Ética de la Facultad de Ciencias Médicas de la Universidad Nacional de Asunción.

\section{ANÁLISIS DE DATOS}

Además de los datos descriptivos de las variables, se utilizó MANOVA con post hoc Duncan y correlación de

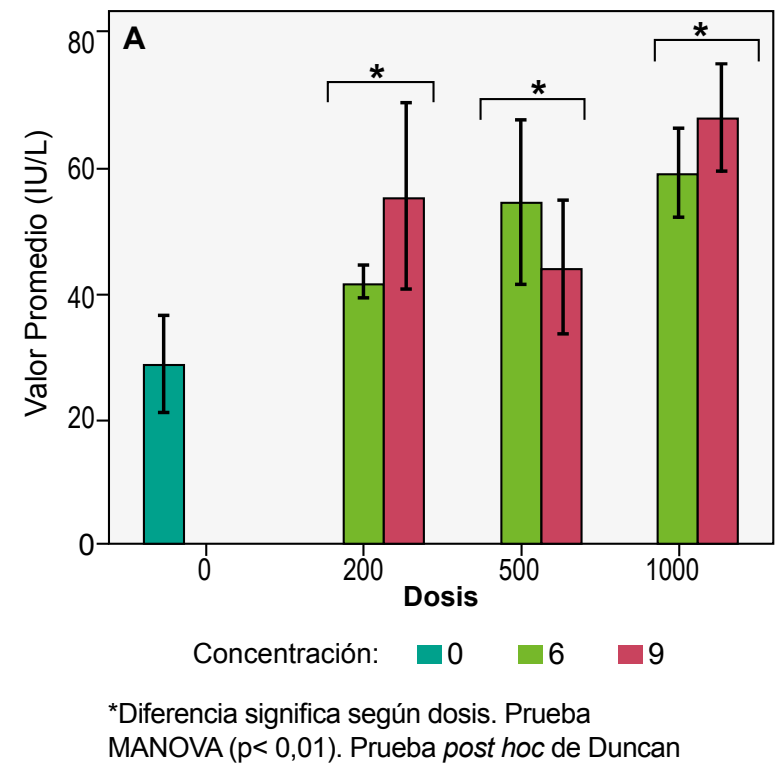

rangos de Pearson, el análisis se realizó con el software SSPS en su versión 15.0. También se añaden cálculos para extrapolar los datos de dosis a la especie humana.

\section{RESULTADOS}

Durante la realización del trabajo no se identificaron diferencias significativas en los parámetros relacionados a la observación diaria. Los resultados de las pruebas laboratoriales mostraron diferencias significativas $(p=0,01)$ entre los niveles de GOT y las dosis administradas del extracto de la planta (Figura 1). Todas las dosis administradas tuvieron valores significativamente mayores al control; entre las dosis administradas, existió diferencia entre $200 \mathrm{mg}$ y $100 \mathrm{mg} ; 500 \mathrm{mg}$ y $100 \mathrm{mg}$. Además, se constató correlación entre la GOT y la dosis de $74 \%$; GOT y concentración de $66 \%$.

En cuanto a urea en sangre, existieron diferencias significativas $(p=0,03)$ entre el grupo control y las concentraciones administradas (Figura 2); sin embargo, no existió diferencia entre las concentraciones de 6 y $9 \%$ del extracto. También se constató correlación del $66 \%$ entre el nivel de urea y dosis; urea y concentración de $79 \%$.

\section{EVALUACIÓN ANATOMOPATOLÓGICA}

En la evaluación macroscópica no se encontraron diferencias significativas $(p>0,05)$. A partir de la dosis de $200 \mathrm{mg} / \mathrm{kg}$ se determinaron modificaciones estructurales en los cordones sinusoidales, tinción granular de los hepatocitos, edema, congestión vascular y esteatosis (Tabla 1). El estudio

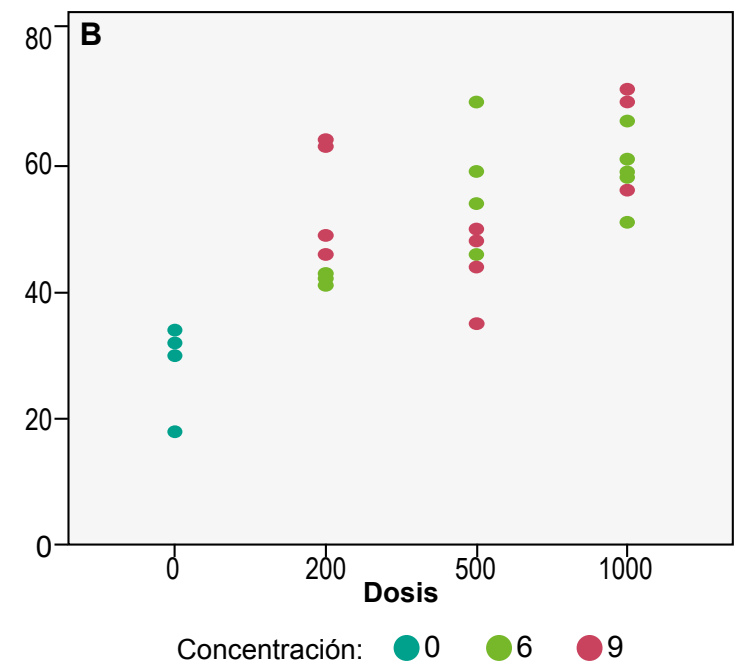

Correlación de Pearson. 0,74 según dosis $(p<0,001)$, 0,66 según concentración $(p<0,01)$

Figura 1. A. Valor promedio de GOT según dosis y concentración de Xanthium spinosum. B. Valores de GOT según dosis y concentración de Xanthium spinosum 


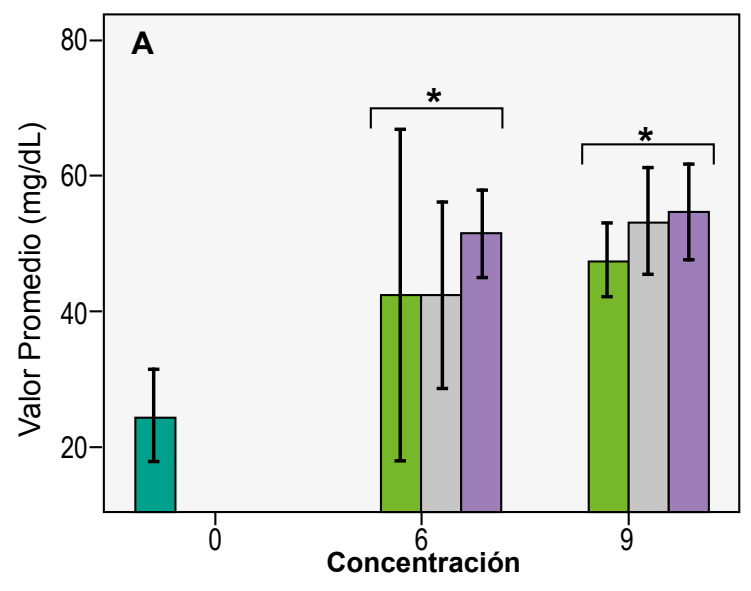

Dosis: $\quad 0 \quad 200 \quad 500 \quad \square 1000$

*Diferencia significativa según concentración.

Prueba MANOVA $(\mathrm{p}<0,01)$. Prueba post hoc de Duncan.

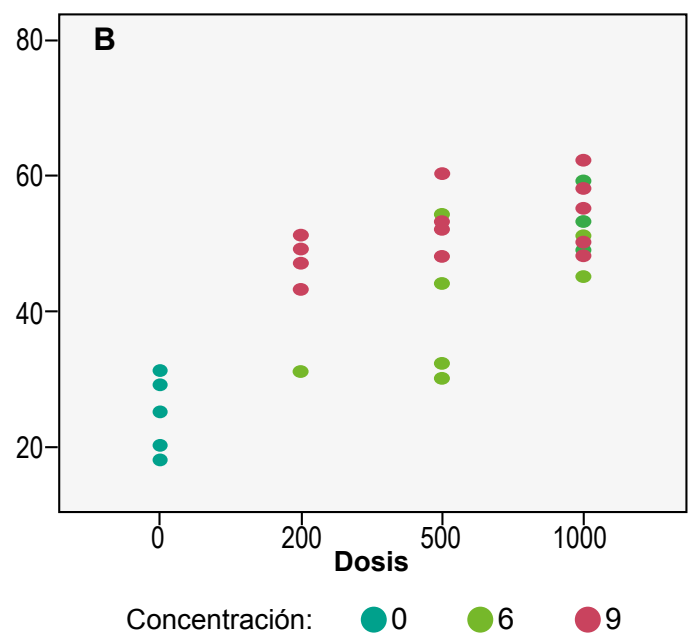

Correlación de Pearson. 0,66 según dosis $(p<0,001), 0,79$ según concentración $(p<0,01)$

Figura 2. A. Valor promedio de urea según dosis y concentración de Xanthium spinosum. B. Valores de urea según dosis y concentración de Xanthium spinosum

anatomopatológico de los órganos pudo constatar mayor esteatosis en el hígado de los ratones que consumieron el extracto de Xanthium spinosum, además de la presencia de edema y tinción granular. No se pudieron corroborar daños en los demás órganos por medio de la técnica de observación utilizada.

\section{EXTRAPOLACIÓN DE DATOS}

Para extrapolar los datos se tiene en cuenta la menor dosis a la cual se encontraron alteraciones $(200 \mathrm{mg} / \mathrm{kg})$. Para estimar la cantidad de hojas que debería de consumirse para reproducir las modificaciones se realizaron los siguientes cálculos: se utilizó aproximadamente $150 \mathrm{~g}$ de hojas secas para la producción de $6 \mathrm{~g}$ de extracto, por lo tanto, el ratón consume $5 \mathrm{~g} / \mathrm{kg}$ de hojas secas en una dosis de $200 \mathrm{mg} / \mathrm{kg}$; al considerar que se pierde aproximadamente el $80 \%$ del peso de las hojas hidratas cuando se secan, el ratón consume $25 \mathrm{~g}$ de hojas hidratadas estimativamente. Extrapolando a un adulto de $60 \mathrm{~kg}$, una dosis de $200 \mathrm{mg} / \mathrm{kg}$ en ratón equivaldría a una dosis de $16,21 \mathrm{mg} / \mathrm{kg}$ en un adulto, por ende, debería consumir $972,6 \mathrm{mg}$ de extracto, equivalente a $24,3 \mathrm{~g}$ de hojas secas o $121,5 \mathrm{~g}$ de hojas hidratadas. Sin embargo, extrapolando a un niño de $10 \mathrm{~kg}$, una dosis de $200 \mathrm{mg} / \mathrm{kg}$ en ratón equivaldría a una dosis de $24 \mathrm{mg} / \mathrm{kg}$ en un niño, por ende, debería consumir 240 $\mathrm{mg}$ de extracto, equivalente a $6 \mathrm{~g}$ de hojas secas o $30 \mathrm{~g}$ de hojas hidratadas (Figura 3 ).

\section{DISCUSIÓN}

Los resultados indican una posible lesión hepática, pues se determinaron diferencias significativas en los niveles de GOT de los grupos que consumieron el extracto en comparación al grupo control $(p<0,01)$, además de una

Tabla 1. Evaluación microscópica de hígado según dosis y concentración de Xanthium spinosum

\begin{tabular}{|c|c|c|c|c|c|c|c|}
\hline \multirow{3}{*}{ Marcador } & \multicolumn{3}{|c|}{ Concentración $6 \%$} & \multicolumn{3}{|c|}{ Concentración 9\% } & \multirow{3}{*}{ Control } \\
\hline & \multicolumn{3}{|c|}{ Dosis } & \multicolumn{3}{|c|}{ Dosis } & \\
\hline & $200 \mathrm{mg}$ & $500 \mathrm{mg}$ & $1000 \mathrm{mg}$ & $200 \mathrm{mg}$ & $500 \mathrm{mg}$ & $1000 \mathrm{mg}$ & \\
\hline Esteatosis* & $25 \%$ & $20 \%$ & $20 \%$ & $10 \%$ & $15 \%$ & $25 \%$ & $5 \%$ \\
\hline Edema & + & + & + & + & + & + & - \\
\hline Tinción granular & + & + & + & + & + & + & - \\
\hline Congestión vascular & + & + & + & + & + & + & + \\
\hline
\end{tabular}

(+) presente; (-) ausente *promedio de cinco campos (40X) donde se observó la lesión 


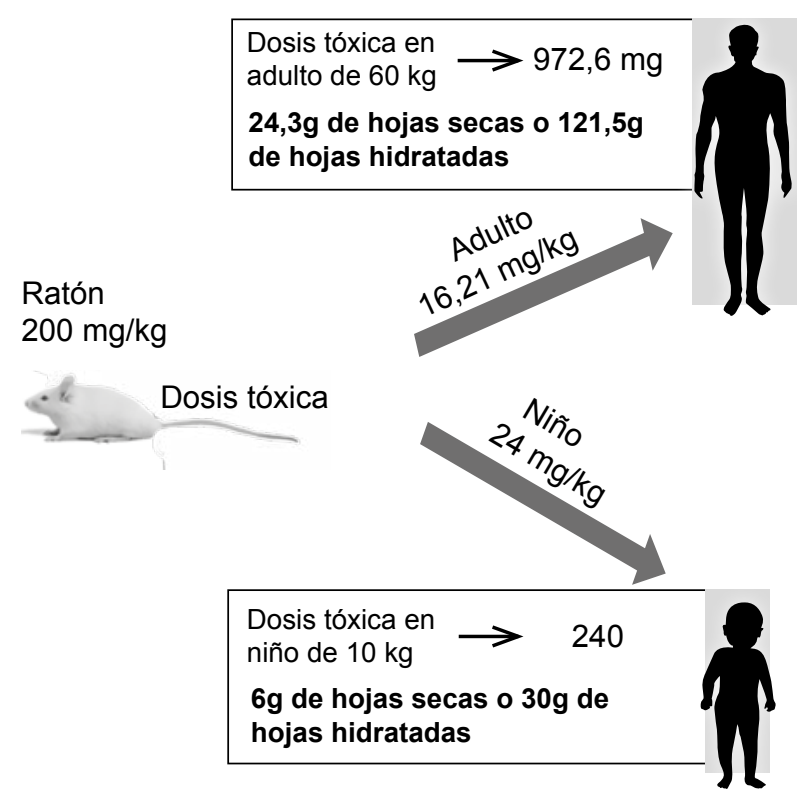

Figura 3. Extrapolación de dosis tóxica del extracto de Xanthium spinosum

correlación positiva entre nivel de GOT y dosis $(0,74)$; GOT y concentración $(0,66)$. En cuanto al examen histológico, el hígado de los grupos que consumieron Xanthium spinosum, presentaron ligero aumento en el porcentaje de esteatosis, además de presencia de edema y tinción granular, que podrían indicar toxicidad.

Estas alteraciones son producidas por los agentes tóxicos de la planta, entre los que se encuentran los glucósidos diterpénicos, como el atractilósido (ATR) y el carboxiatractilósido (CATR), estos son inhibidores específicos del transportador ATP/ADP, presente en la membrana de las mitocondrias. El trasportador ATP/ ADP es fundamental para la fosforilación oxidativa y en consecuencia para mantener los niveles energéticos de la célula, estos inhibidores pueden causar alteraciones celulares principalmente en las células con mayor necesidad energética, como las renales y hepáticas ${ }^{(11)}$. EI CATR posee un grupo carboxilato extra, el cual le confiere una afinidad diez veces mayor que la del ATR, y podría presentar mayor toxicidad en comparación al ATR ${ }^{(12)}$.

Trabajos con otras plantas de la familia Xanthium $s p$. han demostrado patrones de toxicidad similares, aumento de enzimas hepáticas, estrés oxidativo, cambios histológicos, además de alteraciones en el metabolismo de ácidos grasos, aminoácidos e incluso de carbohidratos ${ }^{(11,13,14)}$.

En cuanto a la función renal, los niveles de urea plasmática en los grupos experimento, se encontraron aumentados significativamente según la concentración de Xanthium spinosum administrada $(p<0,01)$, además de una correlación positiva entre nivel de urea plasmática y dosis $(0,66)$, también entre urea y concentración $(0,79)$. Sin embargo, no se pudo constatar lesiones histológicas en los cortes renales.

El ATR también puede provocar alteraciones mitocondriales en las células renales, con un perfil tóxico similar al de la célula hepática, presentando pérdida progresiva de la energía celular y alteraciones metabólicas, en consecuencia, la región renal más afectada suele ser el túbulo proximal ${ }^{(15)}$, estas alteraciones se acompañan de modificaciones en la vasorreactividad de las células musculares de las arteriolas debido a la disminución del ATP ${ }^{(16)}$ y acumulación de ácidos grasos libres en las mitocondrias durante el proceso de hipoxiareoxigenación, lo que podría agravar la toxicidad del compuesto (17).

En el caso de Xanthium strumarium, que posee una proporción similar de glucósidos diterpénicos, algunos artículos proponen que el agente tóxico está presente solamente en las semillas y brotes durante el estadio de cotiledones, y su ausencia en las plantas con cuatro o más hojas ${ }^{(18)}$. Sin embargo, otros estudios aseguran que el extracto de la planta madura también posee una toxicidad importante, produciendo las mismas alteraciones observadas en la toxicidad por ATR y CATR ${ }^{(19)}$. Estos últimos coinciden con los resultados obtenidos con el extracto de hojas de la planta madura de Xanthium spinosum.

Terapias herbales han ganado mucha popularidad entre el público en general, pero en comparación con las terapias aprobadas por las autoridades oficiales, los estudios toxicológicos no se encuentran disponibles. Según los resultados, es necesario $24,3 \mathrm{~g}$ de hojas secas o $121,3 \mathrm{~g}$ de hojas hidratadas para reproducir el patrón toxico en adultos. Se estima que una persona adulta, que consume una planta medicinal de manera tradicional en decocción o infusión, lo hace aproximadamente en una dosis equivalente a $8 \mathrm{~g}$ de material vegetal seco/24 h, de esa manera un adulto de $70 \mathrm{~kg}$ de peso consume aproximadamente una dosis de $0,11 \mathrm{~g}$ de material vegetal seco $/ \mathrm{kg} / \mathrm{di} a$, por tal motivo, el riesgo de intoxicación en adultos es improbable.

Sin embargo, aproximadamente el consumo de $30 \mathrm{~g}$ de hojas hidratas $06 \mathrm{~g}$ de hojas secas de la planta madura de Xanthium spinosum podría causar el mismo patrón de toxicidad en un niño de $10 \mathrm{~kg}$, en consecuencia, los niños e infantes son la población más susceptible a efectos adversos y toxicidad, por las diferencias fisiológicas, sistema metabólico inmaduro y mayor biodisponibilidad a la misma dosis ${ }^{(20)}$. Esto concuerda con lo citado por Alonso J, quien menciona la prohibición de su uso en embarazadas y lactantes, considerando lactantes a niños de hasta dos años o mayores de $10 \mathrm{~kg}{ }^{(21)}$. La importancia de estos 
datos también se reflejan en estudios epidemiológicos sobre intoxicación aguda por Xanthium strumarium, en los que se registra que el $84 \%$ de las muertes se produjo en menores de 15 años ${ }^{(18)}$

La toxicidad de los ATR en hierbas medicinales puede ser reducida por el tratamiento hidrotérmico, por lo tanto, existe la posibilidad de que las moléculas de ATR hayan disminuido por descomposición, hidrólisis y saponificación después de calentar con agua (rotavapor) durante un largo periodo ${ }^{22}$. Sin embargo, la forma de consumo, infusión en frío, en Paraguay no disminuye la toxicidad, por lo que se sugiere más estudios que puedan simular la forma de consumo cultural, además de métodos más sensibles y específicos para detectar alteraciones en otros órganos.

En conclusión, no se percibieron modificaciones físicas, ni de comportamiento. Los marcadores GOT y urea presentaron variaciones, y en cuanto a la histopatología se observaron discretas lesiones que podrían indicar daño tisular en el hígado. Se puede inferir la posibilidad de toxicidad aguda por extracto de hojas de la planta madura de Xanthium spinosum en riñón e hígado; además de considerar la importancia de la dosis y la concentración al momento de la administración, mostrando un interesante comportamiento los cuales pueden llegar a ser diferentes. Tras la extrapolación de dosis al humano, las dosis resultan ser tóxicas y los niños e infantes constituyen una población especialmente susceptible.

Agradecimientos: a la Prof. QF. Rosa Degen por la ayuda en la preparación del extracto. Al Departamento de Medicina Tropical del Instituto de Investigaciones en Ciencias de la Salud de la Universidad Nacional de Asunción, por abrirnos las puertas y acompañarnos en la realización del trabajo.

Contribución de los autoría: ASI, SMG, AMO, MMC han participado de la concepción del trabajo, recolección de datos y aprobación final. ASI y SMG analizaron los datos. AGA y SFT ayudaron a obtener materiales de estudio y asesoraron técnicamente. SIA redactó el artículo y SFT realizo la revisión crítica.

Fuentes de financiamiento: autofinanciado.

Conflictos de interés: los autores declaran no tener conflictos de interés.

\section{REFERENCIAS BIBLIOGRÁFICAS}

1. Cácerers M, Machaín M. Manual de uso de hierbas medicinales del Paraguay. Montevideo; 2001.

2. Ibarrola D, Degen de Arrúa R, ed; Facultad de Ciencias Químicas. Universidad Nacional de Asunción. Catalogo ilustrado de 80 plantas medicinales del Paraguay. Asunción: UNA; 2011.

3. Güez CM, Waczuk EP, Pereira KB, Querol MV, da Rocha JB, de Oliveira LF. In vivo and in vitro genotoxicity studies of aqueous extract of Xanthium spinosum. Braz J Pharm Sci. 2012;48(3):461-7.

4. Hossein Saidi MM. Toxic effect of xanthium strumarium as an herbal medicine preparation. EXCLI Journal. 2009;8:115-7.

5. Witte ST, Osweiler GD, Stahr HM, Mobley G. Cocklebur toxicosis in cattle associated with the consumption of mature Xanthium strumarium. J Vet Diagn Invest. 1990;2(4):263-7.

6. Talakal TS, Dwivedi SK, Sharma SR. In vitro and in vivo antitrypanosomal activity of Xanthium strumarium leaves. J Ethnopharmacol. 1995;49(3):141-5.

7. Bussmann RW, Malca G, Glenn A, Sharon D, Nilsen B, Parris B, et al. Toxicity of medicinal plants used in traditional medicine in Northern Peru. J Ethnopharmacol. 2011;137(1):12140. doi: 10.1016/j.jep.2011.04.071.

8. Fretes F. Plantas medicinales y aromáticas: una alternativa de producción comercial. Paraguay: Agencia del Gobierno de los Estados Unidos para el Desarrollo Internacional (USAID); 2010.

9. Reagan-Shaw S, Nihal M, Ahmad N. Dose translation from animal to human studies revisited. FASEB J. 2008;22(3):659-61.

10. Olfert E, Cross B, McWilliam A ed; Consejo Canadiense de Protección de los Animales. Manual sobre el cuidado y uso de los animales de experimentación. 2 ed. Ottawa: IICA; 1998.

11. Obatomi DK, Bach PH. Biochemistry and toxicology of the diterpenoid glycoside atractyloside. Food Chem Toxicol. 1998;36(4):335-46.

12. Kedrov A, Hellawell AM, Klosin A, Broadhurst RB, Kunji ER, Müller DJ. Probing the interactions of carboxyatractyloside and atractyloside with the yeast mitochondrial ADP/ATP carrier. Structure. 2010;18(1):39-46. doi: 10.1016/j.str.2009.11.009.

13. Wang Y, Han T, Xue LM, Han P, Zhang QY, Huang BK, et al. Hepatotoxicity of kaurene glycosides from Xanthium strumarium L. fruits in mice. Pharmazie. 2011;66(6):445-9.

14. Xue LM, Zhang QY, Han P, Jiang YP, Yan RD, Wang Y, et al. Hepatotoxic constituents and toxicological mechanism of Xanthium strumarium L. fruits. J Ethnopharmacol. 2014;152(2):272-82. doi: 10.1016/j. jep.2013.12.024.

15. Obatomi DK, Bach PH. Atractyloside nephrotoxicity: in vitro studies with suspensions of rat renal fragments and precision-cut cortical slices. In Vitr Mol Toxicol. 2000;13(1):25-36.

16. Song R, Bian H, Huang X, Zhao KS. Atractyloside induces low contractile reaction of arteriolar smooth muscle through mitochondrial damage. J Appl Toxicol. 2012;32(6):402-8. doi: $10.1002 /$ jat. 1688 .

17. Feldkamp T, Kribben A, Roeser NF, Ostrowski T, Weinberg JM. Alleviation of fatty acid and hypoxiareoxygenation-induced proximal tubule deenergization by ADP/ATP carrier inhibition and glutamate. Am J Physiol Renal Physiol. 2007 May;292(5):F1606-16.

18. GurleyES, Rahman M,Hossain MJ,Nahar N, Faiz MA, Islam N, et al. Fatal outbreak 
from consuming Xanthium strumarium seedlings during time of food scarcity in northeastern Bangladesh. PLoS One. 2010;5(3):e9756. doi: 10.1371/ journal.pone.0009756.

19. Islam MR, Uddin MZ, Rahman MS, Tutul E, Rahman MZ, Hassan MA, et al. Ethnobotanical, phytochemical and toxicological studies of Xanthium strumarium L. Bangladesh Med Res
Counc Bull. 2009;35(3):84-90.

20. Tomassoni AJ, Simone K. Herbal medicines for children: an illusion of safety?. Curr Opin Pediatr. 2001;13(2):162-9.

21. Alonso J. Tratado de Fitofármacos y Nutracéuticos. Buenos Aires: Corpus; 2007.

22. Chen LY, $\mathrm{Hu}$ A, Chang CJ. The Degradation Mechanism of Toxic Atractyloside in Herbal Medicines by
Decoction. Molecules. 2013;18(2):201828. doi:10.3390/molecules 18022018 .

Correspondencia: Rubén Arturo Silvero Isidre. Dirección: Facultad de Ciencias Médicas. Universidad Nacional de Asunción. Dr. José Montero c/ Dr. Mario Mazzei. Asunción, Paraguay.

Correo electrónico: arturosilvero@hotmail.com

\section{https://www.rpmesp.ins.gob.pe/}

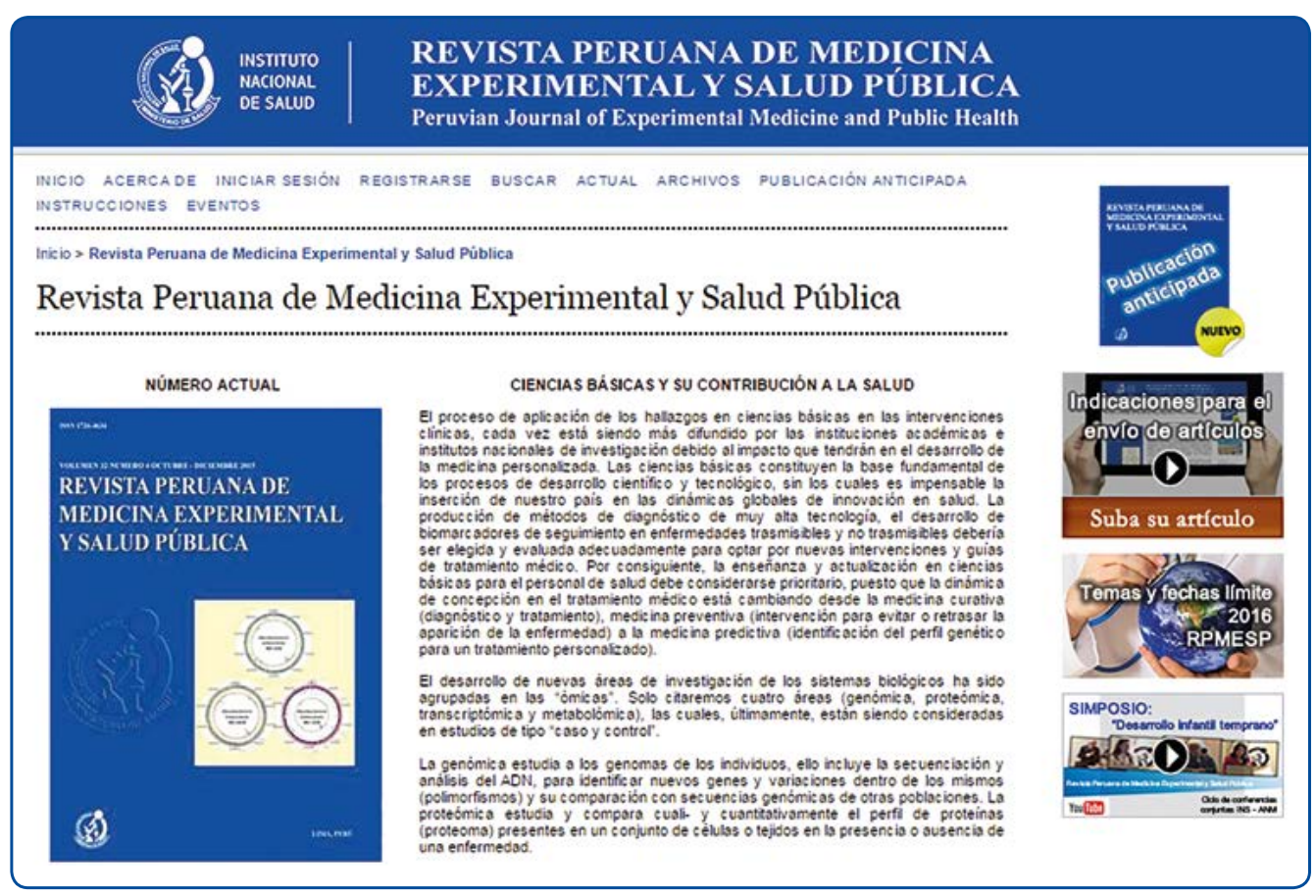

